\title{
Numerical Study on Leading-Edge Receptivity to Freestream Vertical Vorticity*
}

\author{
Yu NISHIO**, Masaya SHIGETA**, Seiichiro IZAWA** and Yu FUKUNISHI ${ }^{* *}$ \\ ** Department of Mechanical Systems and Design, Tohoku University \\ 6-6-01 Aramaki-Aoba, Aoba-ku, Sendai 980-8579, Japan \\ E-mail: nishio@fluid.mech.tohoku.ac.jp
}

\begin{abstract}
Three-dimensional numerical simulation of the flow around a leading edge of a flat plate when vortices with their axes normal to the flat plate surface is carried out to investigate the leading-edge receptivity to the vortical disturbances. It is shown that the vertical vortices outside the boundary layer are not titled and deformed, contradicting to what was reported in previous studies. It is revealed that the streamwise structures, which are dominant in the boundary layer, are formed due to the velocity field induced by the wall-normal vortices outside the boundary layer. It also is found that neighboring pair of vortices side-by-side in the spanwise direction are connected to each other very close to the wall.
\end{abstract}

Key words : Leading Edge Receptivity, Numerical Simulation, Vertical Vorticity

\section{Introduction}

Transition of a boundary layer generally starts from a receptivity process in which the environmental disturbances such as the sound and vorticity enter the boundary layer from the leading edge (Morkovin ${ }^{(1)}$ ). Boundary-layer receptivity has been studied analytically and experimentally for the last several decades. As for the acoustic receptivity, Goldstein ${ }^{(2)(3)}$ analytically discussed the wavelength conversion between the long wavelength freestream acoustic perturbations and the short wavelength TS waves by the asymptotic eigensolutions. This conversion takes place in the nose section, where the surface geometry varies within a short distance. Hammerton and Kerschen ${ }^{(4)(5)}$ showed the nose radius effects on the leadingedge receptivity using an asymptotic method. Haddad et al. ${ }^{(6)(7)}$ simulated a flow field around a parabolic leading edge. They reported that the receptivity becomes higher when nose radius is smaller, especially high for the infinitely sharp leading edge.

Besides acoustic waves, two-dimensional moderate vorticity disturbances also can produce TS waves in the boundary layer (Schubauer et al. ${ }^{(8)}$, Kachanov et al. ${ }^{(9)}$, Kendall ${ }^{(10)}$, Buter et al. $\left.{ }^{(11)}\right)$. When the freestream disturbance becomes larger, streamwise streaks with low frequency and high amplitude starts to appear in the transitional boundary layer. Kendall ${ }^{(12)} \mathrm{ex}-$ perimentally investigated the leading-edge effects on the receptivity to vorticity disturbances. He showed that the aspect ratio of the leading-edge ellipse did not affect the K-mode disturbances in the boundary layer, although the amplitude of T-S waves increased nonlinearly with the decrease in the aspect ratio. Kogan et al. ${ }^{(13)}$ investigated the response of the boundary layer with a blunt nose leading edge to the steady vortices normal to the leading edge. They showed the huge vortex deformation and the flow separation appeared around the stagnation point and at the junction of the leading edge and flat plate. Westin et al. ${ }^{(14)}$ performed a wind tunnel experiment using a jet through a pipe to introduce a three-dimensional localized disturbance near the leading edge. They observed streaky structures similar to those in the boundary layer subjected to the high freestream turbulence (FST). They pointed out that the streamwise vortices were important for the generation of the streaky structures. Goldstein and Wundrow ${ }^{(15)}$ theoretically investigated the effects of vertical vorticity fluctuations on the 
leading-edge receptivity and pointed out the importance of the vortex tilting and stretching by the leading edge. Such a vortex deformation becomes the source of streamwise vorticity in the flow above the flat plate. Also, Goldstein and Leib ${ }^{(16)}$ investigated the receptivity to weak streamwise vorticity and emphasized that the leading-edge effects were insignificant. Wundrow and Goldstein ${ }^{(17)}$ numerically analyzed the effect of steady cross-stream distortion in the upstream flow, or the counter-rotating vortices, on the boundary layer developing on a zero-thickness flat plate. This flow can cause the streamwise velocity profiles to develop distinct shear layers in certain localized spanwise regions, namely the streaky structures.

Nagarajan et al. ${ }^{(18)}$ numerically investigated the effect of the leading-edge bluntness on the bypass transition. They showed that particularly strong vortices were amplified at the blunt leading edge because the vortex stretching induced localized streamwise vortical disturbances in the boundary layer. The wavepacket-like disturbances grew as they convected downstream and eventually broke down to form a turbulent spot. Schrader et al. ${ }^{(19)}$ also discussed the receptivity of a flat plate with an elliptical leading edge by a numerical simulation for the vertical, streamwise and spanwise vorticities. They showed that the vertical and streamwise vortices with a low frequency in the freestream direction triggered the bypass transition and that the spanwise vortices affected the T-S waves. Moreover, they also showed that the blunt leading edge is more receptive than the sharper one for the freestream wall-normal vortices.

As mentioned above, the direct interaction between the freestream vortical disturbance and the leading edge enhances the generation of T-S waves or streaky structures in the downstream boundary layer, because the deformation of vortices such as tilting and stretching take place. The freestream vortices also contribute to the growth of boundary layer indirectly through generation of the wall vortices of opposite sign which results from the no-slip condition. Thus, the object of this study is to investigate the leading-edge receptivity to the periodic flat plate-normal vortices of alternating directions in the freestream, focusing on the vorticity patterns in the boundary layer near the leading edge. Three-dimensional numerical simulation is carried out and the relation between their axis-conversion mechanism and the leading-edge receptivity is discussed in detail.

\section{Numerical Method}

The three-dimensional incompressible Navier-Stokes equations are directly solved by finite difference method using a body-fitted coordinate on a regular grid system. The equations are

$$
\frac{\partial u_{i}}{\partial t}+u_{j} \frac{\partial u_{i}}{\partial x_{j}}=-\frac{\partial p}{\partial x_{i}}+\frac{1}{R e} \frac{\partial^{2} u_{i}}{\partial x_{j} \partial x_{j}},
$$

and

$$
\frac{\partial u_{i}}{\partial x_{i}}=0
$$

where all the variables are non-dimensionalized by the freestream velocity $U_{\infty}$ and the semimajor axis $a$. The third-order upwind difference scheme is used for the convection term written in a gradient form. For the other terms, the second-order central difference scheme is employed. In addition, the multi-directional finite difference scheme is also implemented for the discretization of all terms of the N-S equations. The third-order Adams-Bashforth explicit scheme is applied for the convection term and the Crank-Nicolson implicit scheme is used for the viscous term. The MAC method is employed to solve the pressure and the velocity.

Figure 1 shows the computational domain and the coordinate system. The origin of the Cartesian coordinate system is set at the stagnation point of the leading edge, where $x, y$ and $z$ axes denote the streamwise, vertical and spanwise directions, respectively. The axis ratio of the elliptic leading edge is 5 , which is widely regarded as a typical blunt leading edge. Computational region is carefully determined so that the generation of the upstream boundary does not directly affect the vorticity fluctuation at the leading edge. The flat plate 
length is 4 extending till the end of the computational domain, and the spanwise length of the computation domain is 1.6. The numbers of grid points are 449 in the $\xi$ direction, 193 in the $\eta$ direction, and 16 in the $\zeta$ direction. Computation using a finer grid in which the spanwise grid resolution is four times higher is also carried out. All simulations are performed at a Reynolds number of $R e=U_{\infty} a / v=4.0 \times 10^{4}$.

As for the velocity boundary conditions, the no-slip condition is imposed to the wall and the Neumann condition is prescribed at the outlet boundary. The velocity at the outer elliptic boundaries are fixed at $u=1$ and $v=0$. As for the pressure boundary conditions, the Neumann condition is given at the inlet and the wall, and the Dirichlet condition is employed at the outlets, where the outlet pressure is fixed at unity. The periodic boundary condition is used in the spanwise direction for both velocity and pressure boundary conditions.

A periodic disturbance is added in the freestream as an inlet boundary condition after the steady laminar boundary layer is formed along the wall surface. The disturbance in velocity is given by

$$
u^{\prime}= \begin{cases}A \times D(y) \times \sin (2 \pi f t) \times \sin (2 \pi \beta z) & (-3<y<3) \\ 0 & \text { (otherwise) }\end{cases}
$$

where the non-dimensional frequency $f(=1 / T)=1$, the amplitude $A=0.01$, the spanwise wavenumber $\beta=1$ and $t=0$ indicates the moment when the disturbance is added. The vertical and spanwise components of velocity fluctuations, $v^{\prime}$ and $w^{\prime}$, are zero at the inlet. Smoothing function $D(y)$

$$
D(y)= \begin{cases}0.5 \times\left\{\cos \left(\pi \frac{y-2.5}{2}\right)+1\right\} & (2.5<y<3) \\ 1 & (-2.5<y<2.5) \\ 0.5 \times\left\{\cos \left(\pi \frac{y+2.5}{2}\right)+1\right\} & (-3<y<-2.5)\end{cases}
$$

is used to make the velocity fluctuation zero at $y= \pm 3$, that is, the velocity fluctuation becomes zero at the upper and lower boundaries. Converting to a vorticity fluctuation, the above inlet condition corresponds to $\left(\omega_{x}^{\prime}, \omega_{y}^{\prime}, \omega_{z}^{\prime}\right)=(0, \pi / 1.25,0)$. Except for the near-edge region where the smoothing function $D(y)$ works, the vertical component $\omega_{y}^{\prime}$ plays the dominant role in the freestream vorticity disturbance. It should be noted that the velocity fluctuation given at the inlet is not too strong to trigger a significant nonlinear interaction. The forcing frequency changes the streamwise scale of the vortices relative to the leading edge dimension. In this paper, the forcing frequency is fixed at unity and only this case is investigated.

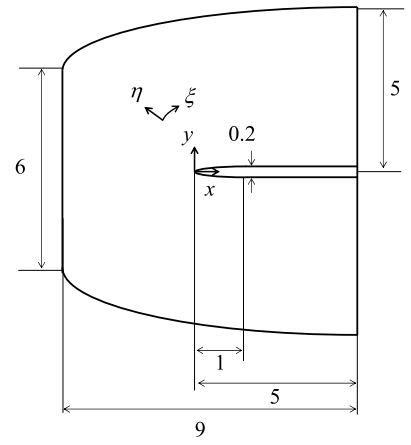

(a) Side view

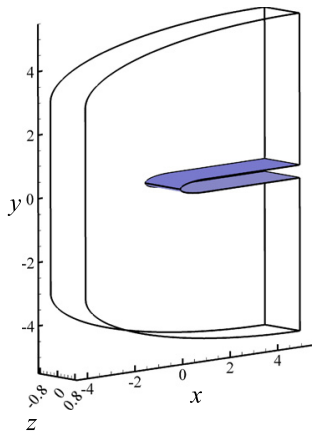

(b) General view

Fig. 1 Computational Domain

\section{Results and Discussion}

\subsection{Mean flow and freestream vorticity disturbance}

Before introducing the vortical disturbances in the freestream, a steady flow over the flat plate with an elliptic leading edge is computed and whether the flow is steady and laminar is 


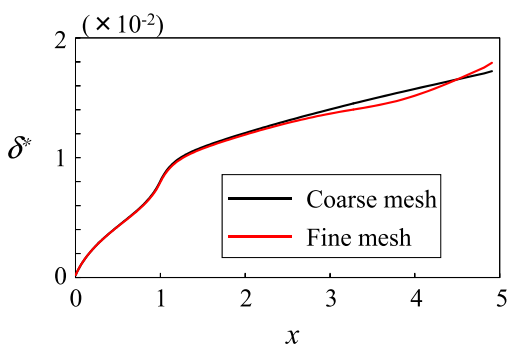

(a) Displacement thickness $\delta^{*}$ obtained from the coarse and fine meshes

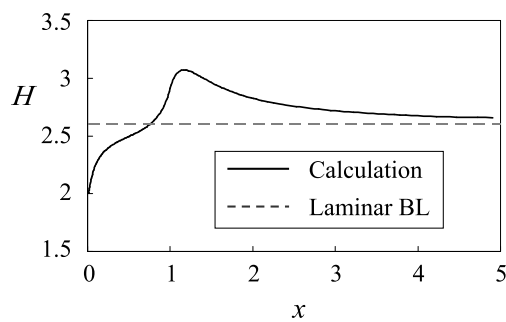

(c) Shape factor

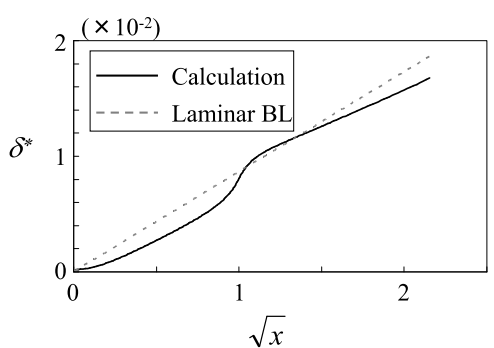

(b) $\delta^{*}$ with respect to $\sqrt{x}$

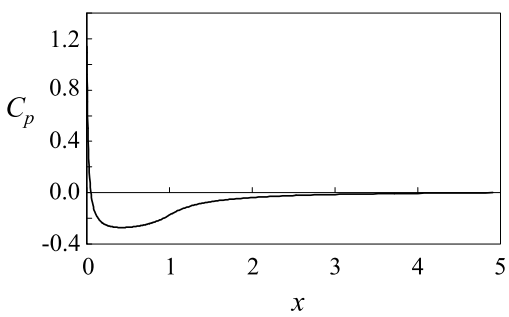

(d) Pressure distribution

Fig. 2 Comparison of the mean flow past the blunt leading edge (-) with the Blasius boundary layer (- - - ).

checked. The displacement thickness $\delta^{*}$ along $x$ is shown in Fig. 2 for the coarse mesh and the fine one. After the rapid increase at the nose section, the displacement thickness gradually increases along the streamwise location. Two curves agree with each other. Figure 2(b) shows the same data as a function of a root of $x$. The displacement thickness $\delta^{*}$ forms a straight line on the flat plate though their slopes are lower than that in the typical Blasius boundary layer. Figure 2(c) shows the variation of the shape factor $H$. The data converge near 2.6, which is the value for the Blasius boundary layer, although it is slightly larger. These discrepancies are likely due to the outer boundary condition above the flat plate. In this simulation, the uniform flow $(u, v, w)=\left(U_{\infty}, 0,0\right)$ is imposed at the upper and lower boundaries; hence, the outer flow is slightly accelerated even though the pressure gradient is almost zero over the flat plate, which is shown in Fig. 2(d). Here, the pressure coefficient $C_{p}$ is obtained by

$$
C_{p}=2 \frac{p-p_{0}}{\rho U_{\infty}^{2}}
$$

The reference pressure $p_{0}$ is chosen to be the value at the outlet boundary, $x=5$. Compared to other simulations, the velocity profiles of the base flows agree well although the computational conditions are slightly different. For instance, the shape factor on the leading edge is larger than the Blasius value of 2.59 and still remains about $2 \%$ larger even downstream of the junction as in Schrader et al. ${ }^{(19)}(\mathrm{AR}=6$ and $\mathrm{Re}=14,400)$, and the pressure coefficient becomes lower than -0.2 near the stagnation point as in Nagarajan et al. ${ }^{(18)}(\mathrm{AR}=6$ and $\mathrm{Re}=57,000)$. Judging from these results, it can be concluded that the boundary conditions used in the current computation have no intrinsic effect on the growth of laminar boundary layer and hence the leading-edge receptivity mechanism. In addition, there are more than twelve grid points in the boundary layer even close to the stagnation point. The velocities measured at the closest points from the wall almost linearly increase. The error from the exact linear increase is less than 0.017. Also, it has been confirmed that the vortex motion is not affected by the grids. So, in the following sections, the calculation is carried out in the coarse mesh.

\subsection{Vorticity component conversion}

From this section, a vorticity disturbance is introduced into the freestream at the inlet boundary. Figures 3 illustrates the vertical and streamwise vorticity distributions in the $x y$ plane at $z=0$. Figure 4 shows the isosurface of $Q$, which is the second invariant of the 
velocity gradient tensor, where red and blue colors indicate whether the vertical vorticity component $\omega_{y}^{\prime}$ is positive or negative. The axes of the introduced vortices are normal to the flat plate surface and their rotating direction changes alternately. The vortices are convected downstream while small portions of the structures are deformed through interaction with the leading edge. It should be noted that the vorticity conversion from the vertical component $\omega_{y}^{\prime}$ to the streamwise component $\omega_{x}^{\prime}$ is limited to a narrow region very close to the wall of the leading edge.

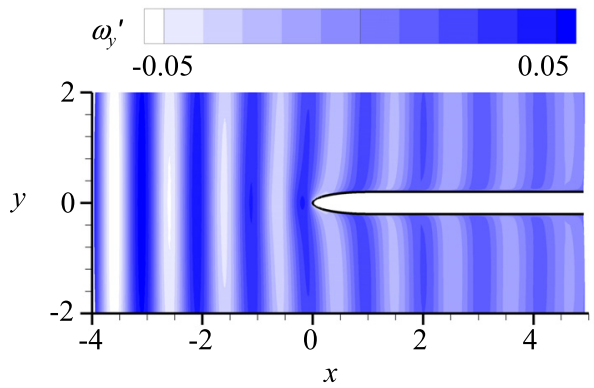

(a) Vertical component $\omega_{y}^{\prime}$

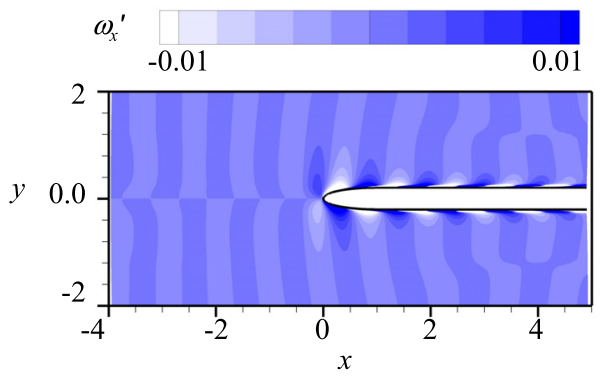

(b) Streamwise $\omega_{x}^{\prime}$

Fig. 3 Vorticity distribution

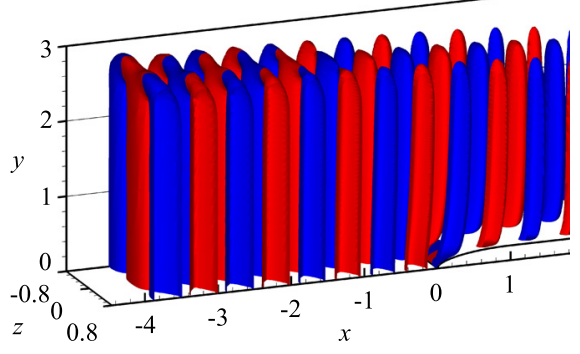

(a) General view

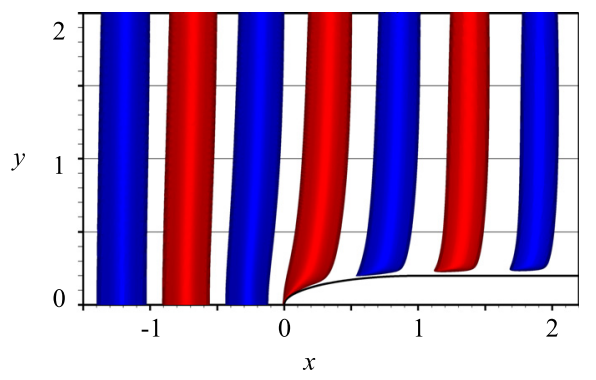

(b) Side view at $z=0$

Fig. 4 Isosurfaces of $Q=5 \times 10^{-5}$

Figure 5 illustrate the isosurfaces of only the positive part of the vertical vorticity $\omega_{y}^{\prime}=$ 0.02 and the streamwise vorticity $\omega_{x}^{\prime}=0.02$. When the vortices hit the leading edge, they are strongly elongated along the wall surface and the streamwise component of vorticity fluctuation appears near the wall surface. These streamwise vorticity regions move downstream with the vortices.

The contour maps of vorticity fluctuation components $\omega_{x, \mathrm{rms}}^{\prime}$ and $\omega_{y, \mathrm{rms}}^{\prime}$ in $x y$ plane at $z=$ 0 are shown in Fig. 6. The intense vertical vorticity fluctuation is observed around the tip of the leading edge, while the streamwise component is widely distributed along the blunt nose.

Figure 7 shows the intensity of the vorticity fluctuation adjacent to the wall surface, measured at various streamwise locations of $z=0$. The streamwise component of vorticity fluctuation $\omega_{x, \mathrm{rms}}^{\prime}$ is found to be much stronger than the vertical component $\omega_{y, \mathrm{rms}}^{\prime}$. It should be noted that both profiles have the points of discontinuity at the same location around $x=$ 1.25. The location coincides to the location where the wall curvature radius abruptly changes at the joint between the elliptical leading edge and the flat plate.

It has been generally believed that longitudinal structures near the wall are generated as a response of the wall to the longitudinal vortices in the freestream and these longitudinal vortical structures produce high and low speed streaky structures near the wall ${ }^{(19)}$. However, in the current result, such longitudinal vortices are never seen in the freestream above the boundary layer. Our results show that the vortices are hardly titled outside the boundary layer, and that the vortex tilting and stretching is limited to a narrow region in the vicinity of the wall as shown in Fig. 4. 


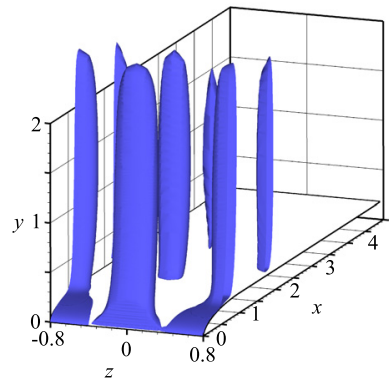

(a-1) $t / T=11.5$

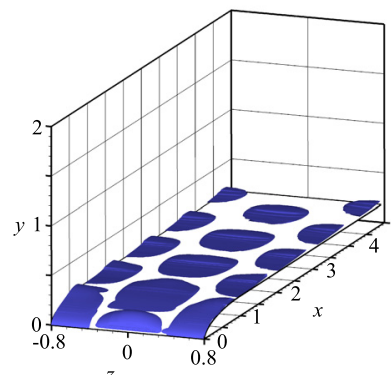

(b-1) $t / T=11.5$

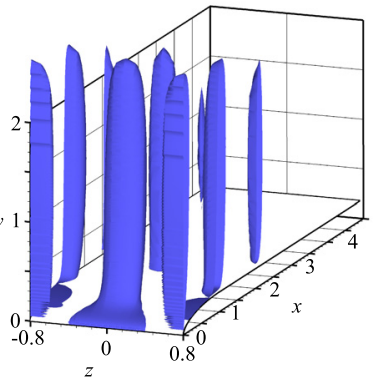

(a-2) $t / T=11.75$

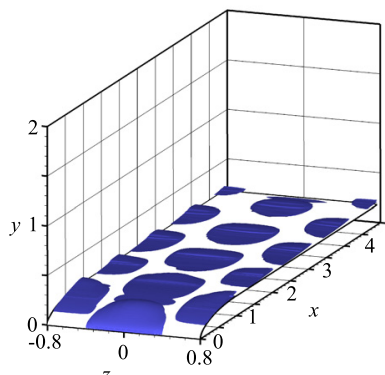

(b-2) $t / T=11.75$

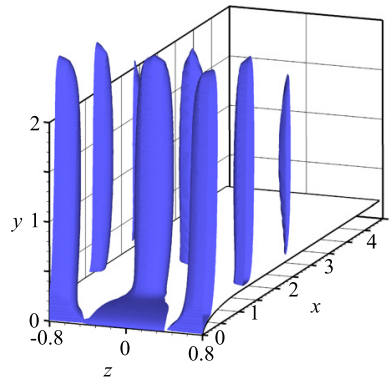

(a-3) $t / T=12.00$

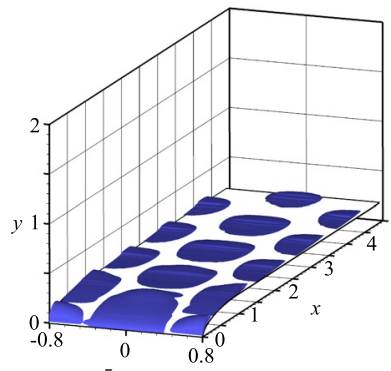

(b-3) $t / T=12.00$

Fig. 5 Vorticity distribution of (a) $\omega_{y}^{\prime}$ and (b) $\omega_{x}^{\prime}$
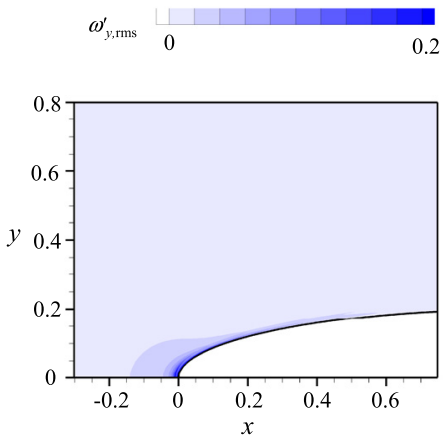

(a) Vertical component of vorticity $\omega_{y}^{\prime}$
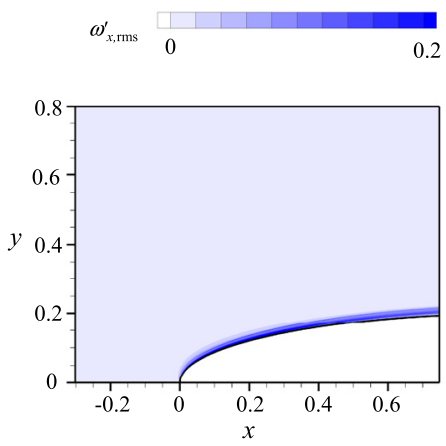

(b) Streamwise component of vorticity $\omega_{x}^{\prime}$

Fig. 6 Vorticity fluctuations around the leading edge at $z=0$

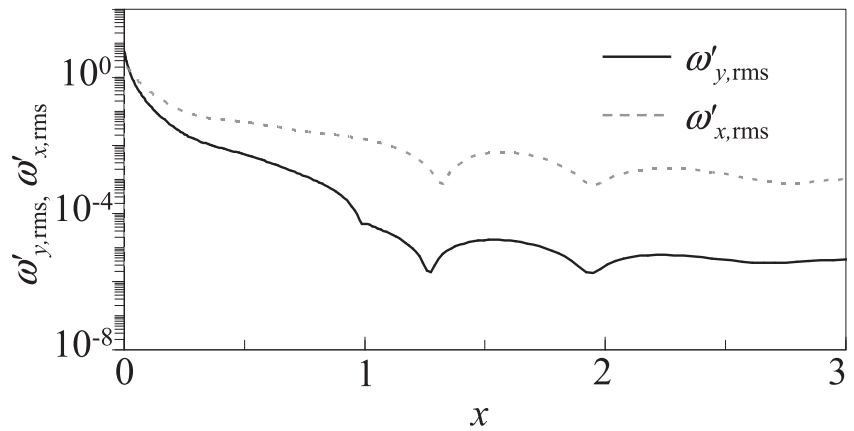

Fig. 7 Streamwise distribution of vorticity fluctuation adjacent to the wall surface at $z=0$ 


\subsection{Streamwise vorticity near the wall}

Figure 8 illustrates the two possible scenarios which may produce the streamwise vorticity components near the wall. They are:

(i) Tilting and stretching of the near-wall part of the preceding vortex (Scenario (i)).

(ii) Vorticity generated on the plate surface because of the no-slip condition, due to velocity induced by the wall-normal vortices (Scenario (ii)).

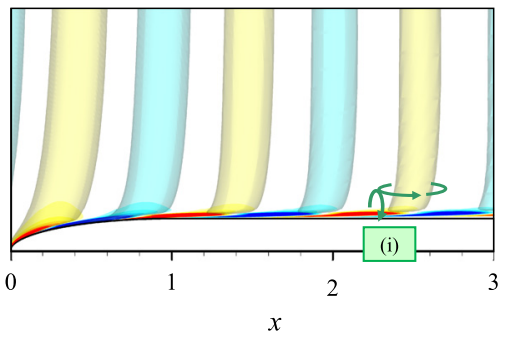

(a) Extension of the preceding vortex (Scenario (i))

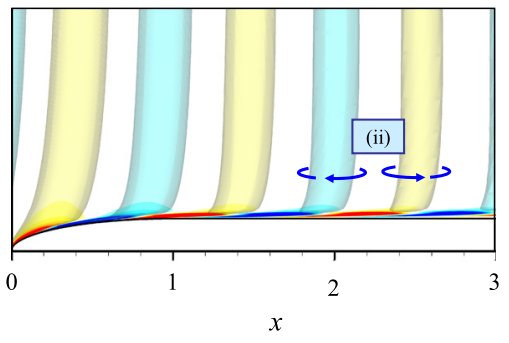

(b) Spanwise velocity induced by the wall-normal vortices (Scenario (ii))

Fig. 8 Illustration of two possible scenarios to produce the streamwise vorticity near the wall

To eliminate the influence of the preceding vortex, only a single vortex is introduced into the freestream, instead of periodic forcing. Velocity fluctuation written in Eq.3 is given at the inlet for only a half period. Figure 9 shows the vorticity fluctuation pattern of $\omega_{y}^{\prime}$ in $x y$ plane at $z=0$ when the negative freestream vortex reaches at $x=2$. In the vicinity of the wall, the positive streamwise fluctuation is observed on its upstream side as well as the downstream negative fluctuation. Figure 10 illustrates the contour map of $\omega_{x}^{\prime}$ together with the velocity vectors in the cross section at $x=1.75$ and 2.25. Both profiles are quite similar to each other except for their signs. This fact exhibits that the streamwise vorticity fluctuation close to the wall can be generated without the preceding vortex. Hence, the scenario (i) can be ruled out.

Figure 11 shows the vorticity fluctuation in the boundary layer at $z=0$. The vorticity fluctuation profile for the periodic forcing case is roughly equal to the addition of the two single vortex cases, at $x=1.75$ and 2.25 .

From these results, it can be concluded that scenario (ii) is likely to be correct explanation. The streamwise vorticities on the wall are mainly generated to satisfy the no-slip condition in response to the spanwise velocity which is induced by wall-normal vortices.

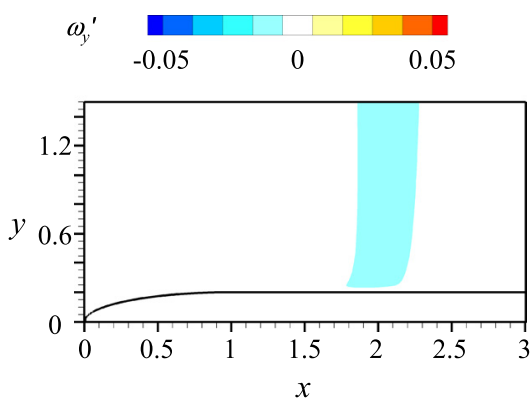

(a) Vertical vorticity fluctuation $\omega_{y}^{\prime}$

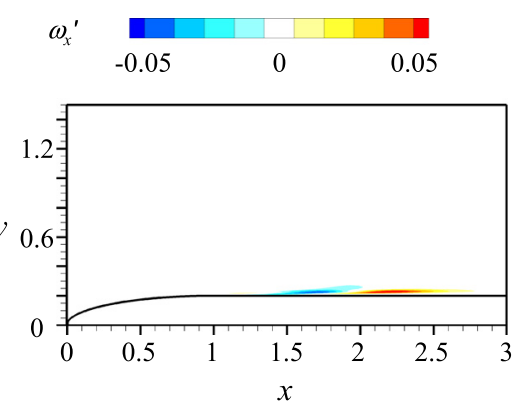

(b) Streamwise vorticity fluctuation $\omega_{x}^{\prime}$

Fig. 9 Vorticity pattern of $\omega_{x}^{\prime}$ and $\omega_{y}^{\prime}$ in $x y$ plane at $z=0$ when the freestream negative vortex reaches at $x=2$

\subsection{Phenomenon at the leading edge}

The phenomenon taking place in the vicinity of the leading edge is closely examined. Figure 12 illustrates the vorticity pattern in the freestream and vorticity pattern generated at the surface of the leading edge. The velocity vectors are also drawn in the figure. The vorticity 


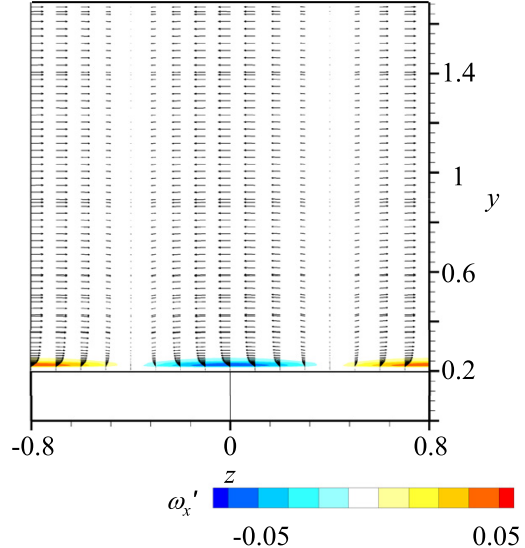

(a) $x=1.75$

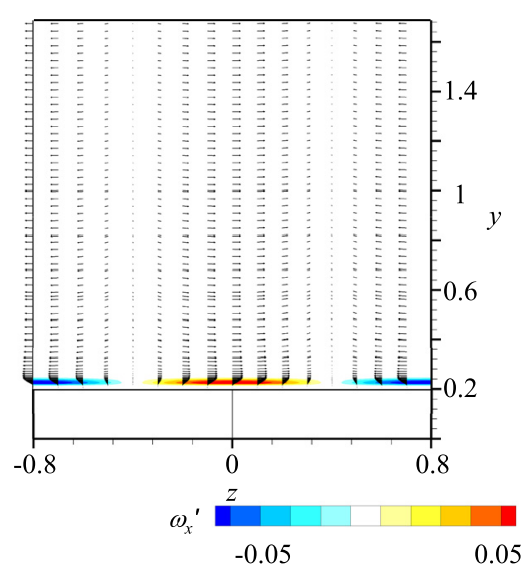

(b) $x=2.25$

Fig. 10 Vorticity pattern of $\omega_{x}^{\prime}$ in $y z$ plane at $x=1.75$ (a) and 2.25 (b) in Fig. 9

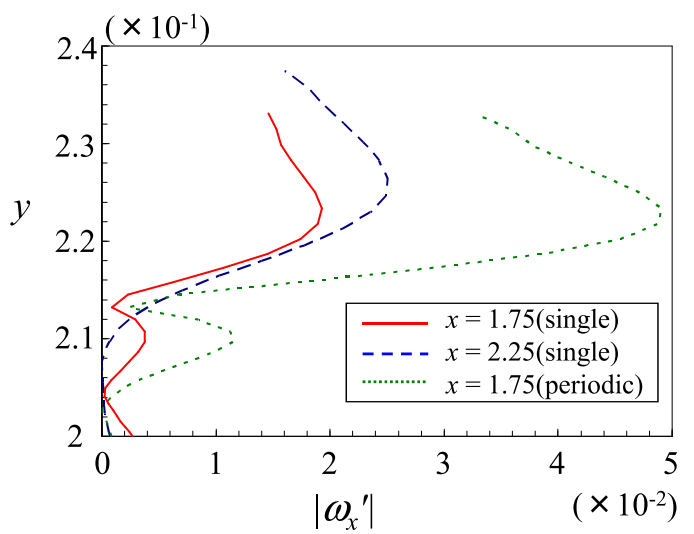

Fig. 11 Comparison of vorticity fluctuation $\omega_{x}^{\prime}$ in the boundary layer between the periodic and the half-period forcing when the freestream negative vortex reaches at $x=2$

regions are generated at the wall surface before the freestream vortices touch the leading edge. Since the velocity must be zero at the wall surface satisfying the no-slip boundary condition despite the velocity induced by the oncoming vortex, the vorticity with the sign opposite of the vortex is generated at the wall surface. After the freestream vortex hits the leading edge, its near-wall part is deformed by tilting in the streamwise direction and spreading in the spanwise direction, as shown in Fig. 13(a). The figure shows the isosurface of $Q=5 \times 10^{-5}$ colored by the sign of local $y$ component vorticity. The red region indicate where $\omega_{y}^{\prime}$ is positive while the blue area is negative. Figure 13(b) shows the isosurfaces of the spanwise vorticity components $\omega_{z}^{\prime}=0.02$ (red) and $\omega_{z}^{\prime}=-0.02$ (blue). The near-wall $Q$ region in Fig 13(a) coincides with the high spanwise vorticity region in Fig. 13(b). Thus, it is found that neighboring pair of vortices side-by-side in the spanwise direction are connected to each other very close the wall. These spanwise structures are soon elongated by the intense streamwise velocity gradient, and they do not appear to play any significant role in the growth of vorticity fluctuations downstream.

\section{Conclusions}

The receptivity of elliptic leading edge to periodic vortices with their axes normal to the flat plate was studied by three-dimensional numerical simulation. The vortices kept their axes normal to the flat plate even after they hit the leading edge. Tilting and stretching of the vortices accompanying the collision were observed only in the vicinity of the wall, contradicting to the previous reports. The streamwise vorticity components inside the boundary layer were generated because of the velocity field induced by the vortices in the freestream, and were 


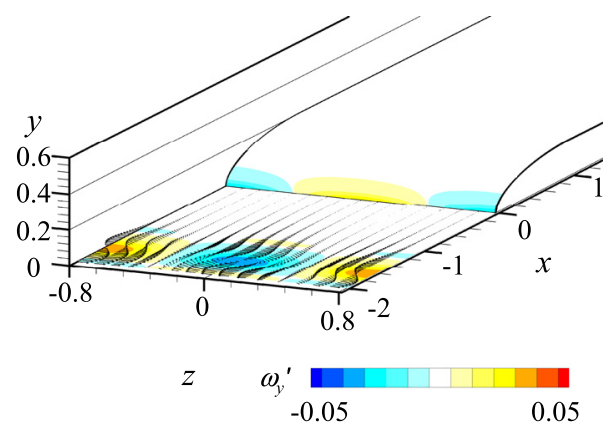

Fig. 12 Distribution of vorticity fluctuation $\omega_{y}^{\prime}$ and velocity vectors in $x z$ plane at $y=$ $0 . \omega_{y}^{\prime}$ adjacent to the wall surface is also shown

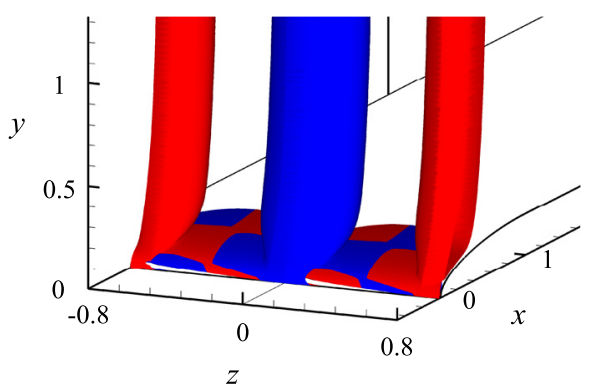

(a) Isosurface of $Q=5 \times 10^{-5}$ colored by the sign of local $y$ component vorticity

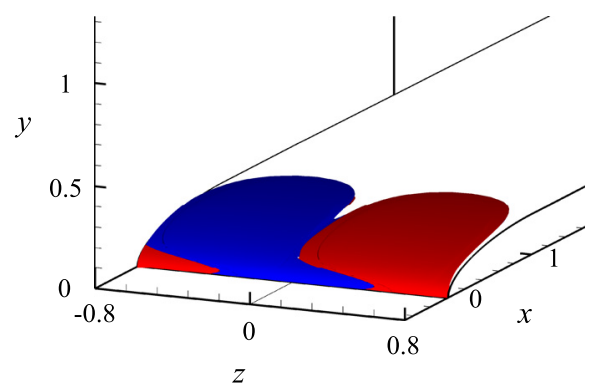

(b) Isosurfaces of the spanwise vorticity components $\omega_{z}^{\prime}=0.02$ (red) and $\omega_{z}^{\prime}=-0.02$ (blue)

Fig. 13 Deformation of freestream vertical vortices and generation of spanwise vorticity at the leading edge

not the extensions of the vortices in the freestream. It was also found that neighboring pair of vortices side-by-side in the spanwise direction were connected to each other very close to the wall. These spanwise structures were soon elongated by the intense streamwise velocity gradient, and they did not appear to play any significant role in the growth of vorticity fluctuations downstream. Investigation of the leading edge area, focusing on the mechanism where the structures inside the boundary layer were generated by the freestream vortices was carried out in this study. Whether the generated structures survive until the downstream location where the fluctuations start to grow and how they affect the transition further downstream is a subject to be studied in the future.

\section{References}

(1) Morkovin, M. V., On the many faces of transition, Viscous Drag Reduction ed. C.S.Wells, (1969), pp.1-31, New York:Plenum.

( 2 ) Goldstein, M. E., The evolution of Tollmien-Schlichting waves near a leading edge, Journal of Fluid Mechanics, Vol.127(1983), pp.59-81.

( 3 ) Goldstein, M. E., Scattering of acoustic waves into Tollmien-Schlichting waves by small streamwise variations in surface geometry, Journal of Fluid Mechanics, Vol.154(1985), pp.509-529.

( 4 ) Hammerton, P. W. and Kerschen, E. J., Boundary-layer receptivity for a parabolic leading edge, Journal of Fluid Mechanics, Vol.31(1996), pp.243-267.

( 5 ) Hammerton, P. W. and Kerschen, E. J., Boundary-layer receptivity for a parabolic leading edge. Part 2. The small Strouhal number limit., Journal of Fluid Mechanics, Vol.353(1997), pp.205-225.

( 6 ) Haddad, O. M. and Corke, T. C., Boundary-layer receptivity to freestream sound on parabolic bodies, Journal of Fluid Mechanics, Vol.368(1998), pp.1-26.

( 7 ) Haddad, O. M. Erturk, E. and Corke, T.C., Acoustic receptivity of the boundary layer 
over parabolic bodies at angles of attack, Journal of Fluid Mechanics, Vol.536(2005), pp. 377-400.

( 8 ) Schubauer, G. B., and Skramstad, H. K., Laminar-Boundary-Layer Oscillations and Transition on a Flat Plate, Journal of Research of the National Bureau of Standards, Vol.38, No.2(1947), pp. 251-292.

( 9 ) Kachanov, Y. S., Kozlov, V. V. and Levchenko, V. Y., Occurrence of TollmienSchlichting waves in the boundary layer under the effect of external perturbations, Fluid Dynamics, Vol.13, No.5(1978), pp.704-711.

(10) Kendall, J. M., Studies on laminar boundary-layer receptivity to freestream turbulence near a leading edge, Proceedings of ASME and JSME Joint Fluids Engineering Conference, No.FED-114(1991-6), pp.23-30.

(11) Buter, T. A. and Reed, H. L., Boundary layer receptivity to free-stream vorticity, Physics of Fluids, Vol.6, No.10(1994), pp.3368-3379.

(12) Kendall, J. M., Boundary layer receptivity to freestream turbulence, AIAA paper, No.901504(1990), pp.1-7.

(13) Kogan, M. N., Shumilkin, V. G., Ustinov, M. V. and Zhigulev, S. V., Response of boundary layer flow to vortices normal to the leading edge, European Journal of Mechanics B/fluids, Vol.20, No.6(2011), pp.813-820.

(14) Westin, K. J. A., Bakchinov, A. A., Kozlov, V. V. and Alfredsson, P. H., Experiments on localized disturbances in a flat plate boundary layer Part 1 . The receptivity and evolution of a localized free stream disturbance, European Journal of Mechanics B/Fluid, Vol.17, No.6(1998), pp.823-846.

(15) Goldstein, M. E. and Wundrow, D. W., On the environmental realizability of algebraically growing disturbances and their relation to Klebanoff mode, Theoretical and Computational Fluid Dynamics, Vol.10(1998), pp.171-186.

(16) Goldstein, M. E. and Leib, S.J., Three-dimensional boundary layer instability and separation induced by small-amplitude streamwise vorticity in the upstream flow, Journal of Fluid Mechanics, Vol.246(1993), pp. 21-41.

(17) Wundrow, D. W. and Goldstein, M. E., Effect on a laminar boundary layer of smallamplitude streamwise vorticity in the upstream flow, Journal of Fluid Mechanics, Vol.426(2001), pp.229-262.

(18) Nagarajan, S., Lele, S. K. and Ferziger, J. H., Leading edge effects in bypass transition, Journal of Fluid Mechanics, Vol.572(2007), pp.471-504.

(19) Schrader, L.-U., Brandt, L., Mavriplis, C. and Henningson, D. S., Receptivity to freestream vorticity of flow past a flat plate with elliptic leading edge, Journal of Fluid Mechanics, Vol.653(2010), pp.245-271. 